\section{Pulmonale Trichomoniasis: Diagnose durch Erregernachweis in der bronchoalveolären Lavage}

Zusammenfassung: Trichomonadeninfektionen des Respirationstraktes wurden in Einzelfällen beschrieben, bei denen zumeist eine pulmonale Grunderkrankung mit Beeinträchtigung des Immunsystems (z.B. Lungenkarzinom, Lungenabszeß, Bronchiektasen) vorlag. Die pulmonale Trichomoniasis wird überwiegend durch Trichomonas tenax, einen normalerweise apathogenen Kommensalen der Mundhöhle verursacht, seltener auch durch andere Trichomonasarten. Eine 45jährige Frau stellte sich wegen zunehmendem trockenen Husten, Belastungsdyspnoe und Abgeschlagenheit vor. Die Beschwerden persistierten über 6 Monate ohne Besserung durch antientzündliche und antiobstruktive inhalative Therapie. Die Patientin hatte vor ca. 20 Jahren eine Sarkoidose der Lunge durchgemacht, die seither asymptomatisch verlaufen war. Andere pulmonale oder extrapulmonale Vorerkrankungen waren nicht aufgetreten. Die Untersuchungen ergaben einen erhöhten ACESpiegel $(90 \mathrm{IE} / \mathrm{ml})$ und eine bronchiale Hyperreagibilität im Histaminprovokationstest bei sonst unauffälligen Befunden (Röntgenbild des Thorax, Bronchoskopie, Lungenfunktionsprüfung, Blutbild und Serumkalzium). Als Ursache der Symptomatik konnte schließlich eine pulmonale Trichomoniasis durch den direkten zytologischen Nachweis zahlreicher Trophozoiten von Trichomonas tenax in der BAL diagnostiziert werden. Während einer Behandlung mit Metronidazol über 40 Tage kam es zur vollständigen Rückbildung der Beschwerden und zu einer Normalisierung des ACE-Spiegels im Serum. Die Patientin ist seit 12 Monaten beschwerdefrei. Die Pathogenität der Infektion mit Trichomonas tenax bei immunkompetenten Patienten ist unklar. Im Vergleich zu den bekannten Fällen wies die Patientin keine der angeführten Risikofaktoren auf. Der Fall zeigt die klinische Relevanz einer pulmonalen Trichomonadeninfektion bei einer ansonsten gesunden Person.

Pulmonary trichomoniasis: Diagnosis based on identification of trichomonads in bronchoalveolar lavage fluid: Bronchopulmonary infections caused by trichomonads have been reported mainly in patients with pre-existing pulmonary or debilitating disease (e.g. bronchial carcinoma, lung abscess, bronchiectasis). Pulmonary trichomoniasis is most often due to infection with Trichomonas tenax, usually regarded as a harmless commensal of the human mouth, and may rarely be caused by other trichomonas species. A 45 year old female presented with a dry cough, exertional dyspnoea and malaise. These symptoms persisted for 6 months regardless of anti-inflamma-

Pneumologie 53 (1999) 617-619

(C) Georg Thieme Verlag Stuttgart · New York
D. F. Stratakis' ${ }^{1}$, S. M. Lang ${ }^{1}$, S. Eichenlaub², T. Löscher², R. Stein ${ }^{3}$, R. M. Huber ${ }^{1}$

1 Schwerpunkt Pneumologie und

${ }^{2}$ Abteilung für Infektions- und Tropenmedizin der Medizinischen Klinik, Klinikum Innenstadt, Ludwig-Maximilians-Universität München, München, Deutschland

${ }^{3}$ Pneumologe, Bad Tölz

tory and anti-obstructive inhalative therapy. Sarcoidosis of the lungs, diagnosed 20 years prior, had been asymptomatic since and there was no coexistent disease. Laboratory data revealed increased ACE-levels $(90 \mathrm{IE} / \mathrm{ml})$ and lung function showed bronchial hyperreactivity on histamine challenge. No other abnormalities were found (chest $\mathrm{x}$-ray, bronchoscopy, lung function test, blood count and serum calcium). The diagnosis was based on the cytological identification of numerous trophozoites of $\mathrm{T}$. tenax in the bronchoalveolar lavage. Therapy with oral metronidazol for $\mathbf{4 0}$ days led to complete recovery from symptoms and normalisation of ACE serum levels. The patient has remained well for 12 months since. The pathogenicity of oral trichomonads in the non-immunocompromised host remains uncertain. Our patient had no known medical risk factors by comparison with published cases. The case illustrates the clinical relevance of pulmonary trichomoniasis in an otherwise healthy person.

\section{Einleitung}

Trichomonaden können in seltenen Fällen zu Infektionen der Atemorgane führen. Die pulmonale Trichomoniasis manifestiert sich klinisch als Infiltrat, Lungenabszeß oder Pleuraerguß/-empyem [1 -9]. Systematische Untersuchungen zur Inzidenz dieses seltenen Krankheitsbildes liegen nicht vor. Die überwiegende Mehrheit der publizierten Fälle klinisch relevanter pulmonaler Infektionen mit Trichomonaden trat bei Immunsupprimierten (Akoholismus, Karzinome) oder bei Bronchiektasen auf $[1,6]$. Trotz systematischer Suche fanden sich in der deutschsprachigen Literatur keine Hinweise auf diese Protozoenerkrankung im Bereich der Atemwege. Vereinzelte Fälle wurden aus verschiedenen geographischen Gebieten berichtet, eine größere Anzahl von Fällen (19 von 112 Patienten, $17 \%$ ) wurden in einer russischen Studie zusammengetragen $[1,10]$. Im Gegensatz zur genitourethralen Trichomoniasis ist der Erreger der pulmonalen Affektion in der Regel nicht Trichomonas vaginalis. Die Infektion wird überwiegend durch Trichomonas tenax hervorgerufen [1,3]. Dieser Erreger kommt als harmloser Saprophyt in der Mundhöhle vor. Eine Assoziation von Infektion und mangelnder enoraler Hygiene wurde wiederholt beschrieben [11,12].

\section{Fallbericht}

Eine 45jährige Frau stellte sich beim Hausarzt wegen zunehmendem trockenen Husten, Belastungsdyspnoe und Abgeschlagenheit vor. Die Patientin war beruflich auf einer Intensivstation in einer oberbayerischen Kleinstadt tätig, war nie 
im Ausland gewesen, Nichtraucherin und gab einen maßvollen Alkoholkonsum an. Ihre Mundhygiene war gut, sie war regelmäßig beim Zahnarzt gewesen. Medikamente, die das Immmunsystem beeinträchtigen, wurden nicht eingenommen. Aus der Vorgeschichte ist erwähnenswert, daß die Patientin vor ca. 20 Jahren eine Sarkoidose der Lunge durchgemacht hatte, die nachfolgend ohne Therapie asymptomatisch verlaufen war. Weitere Erkrankungen der Lunge oder extrapulmonale Erkrankungen wurden nicht angegeben. Aufgrund einer Progredienz der Beschwerden wurde die Patientin an einen Pneumologen zur fachärztlichen Diagnostik und Therapie überwiesen.

- Ein Hinweis auf eine extrapulmonale Manifestation der Sarkoidose ergab sich nicht.

- Das Röntgenbild des Thorax zeigte einen altersentsprechend unauffälligen Befund, insbesondere keine pneumonischen Infiltrate, Ergüsse oder bihiläre Lymphome.

- Laborchemisch zeigte sich lediglich ein erhöhter ACESpiegel im Serum (90 IE/ml), Serumkalzium und Blutbild lagen im Normbereich.

- In der Lungenfunktion zeigte sich bodyplethysmographisch keine Restriktion (TLC 133\% Soll) und keine Obstruktion (Rtot 0.13 kPa*s/l, FEV1 95\% Soll). Nach Provokation mit 1,5 mg Histamin kam es zu einem Abfall des FEV1 von 95\% auf $71 \%$ des Sollwertes als Ausdruck einer bronchialen Hyperreagibilität. Die Diffusionskapazität war nicht eingeschränkt (TLCO in $\mathrm{mmol} / \mathrm{min} / \mathrm{kPa}$ [single-breath] $100 \%$ Soll).

Nachdem initial bereits eine Antibiose mit einem Breitspektrumpenicillin ohne Erfolg war und eine dreimonatige inhalative antientzündliche und bronchodilatorische Therapie $\mathrm{zu}$ keiner Besserung der Symptomatik, bei unverändertem Röntgenbefund, führte, wurde eine bronchoalveoläre Lavage unter dem Verdacht auf ein Rezidiv der Sarkoidose durchgeführt.

- Die Bronchoskopie ergab keine morphologischen Veränderungen.

- In der bronchoalveoären Lavage fand sich eine geringe Vermehrung der Lymphozyten (14\%) sowie multiple Protozoen, die morphologisch als Trichomonas tenax identifiziert wurden (Abb. 1).

\section{Therapie und Verlauf}

Metronidazol, $3 \times 400 \mathrm{mg}$ oral per die, über 40 Tage verabreicht, führte zur vollständigen Beschwerdefreiheit der Patientin und zur Normalisierung der erhöhten ACE-Spiegel im Serum. Nach Absetzen der Therapie blieb die Patientin rezidivfrei über nunmehr 12 Monate.

\section{Diskussion}

Die Diagnose dieser seltenen Protozoenerkrankung der Atemwege wurde im vorliegenden Fall neben dem klinischen Verlauf durch die bronchoalveoläre Lavage erbracht, die den direkten morphologischen Erregernachweis ermöglichte. Der klinische Verlauf war nach 6monatigen Beschwerden durch ein promptes Ansprechen, sowie Rezidivfreiheit nach antiparasitärer Therapie gekennzeichnet. Die klinischen Manifestationen der pulmonalen Trichomoniasis reichen von Zeichen der Hyperreagibilität bis zu radiologisch nachweisbaren Infiltraten und Ergüssen und sind somit von anderen bronchopulmonalen Infekten nicht zu unterscheiden. Laborchemisch zeigt sich häufig eine Eosinophilie, die differentialdiagnostisch an ein exogen-allergisches Asthma bronchiale denken läßt, aber im vorliegenden Fall nicht nachweisbar war [2]. In den seit 1942 beschriebenen Fällen lag zumeist eine pulmonale Grunderkrankung sowie eine Beeinträchtigung des Immunsystems (z.B. Bronchialkarzinom, Lungenabszeß, Bronchiektasen) vor [1-9]. Die Pathogenität der Erreger, d.h. Trichomonas tenax, ist bei immunkompetenten Patienten unklar, über chronische Tonsillitis [12] und Befall einer Speicheldrüse wurde berichtet [13]. Eine Assoziation mit erhöhten ACE-Spiegeln im Serum oder Granulombildung ist aus der englischsprachigen Literatur nicht bekannt. Die Infektion erfolgt in der Regel durch Aspiration von Schleim mit Trichomonaden, die als harmlose Kommensalen die Mundhöhle besiedeln. Die enorale Besiedelung ist in der Regel nicht häufig und wird in der Literatur zwischen 4 und bis zu $53 \%$ angegeben $[1,14,15]$. Trichomonadeninfektionen der Atemwege wurden als Einzelfälle beschrieben, eine größere Population mit pulmonaler Infektion wurde bisher lediglich aus Rußland (19 Fälle) berichtet [10]. Im Vergleich zu den

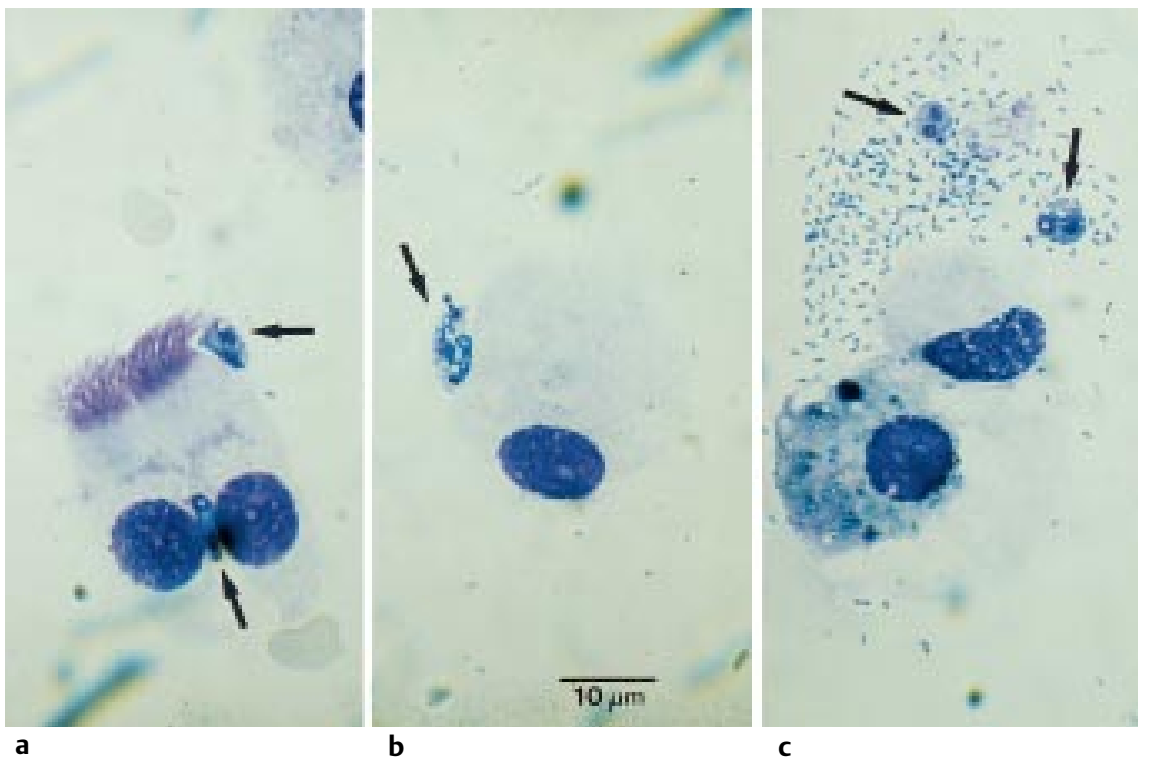

Abb. 1 Trophozoiten von Trichomonas tenax im zytologischen Präparat (May-Grünwald-Giemsa-Färbung) der BAL

a) Trophozoiten (Pfeil) mit respiratorischem Epithel

b) Trophozoiten (Pfeil) mit Alveolarmakrophage

c) Alveolarmakrophagen, Trophozoiten (Pfeil) in einem Bakterienhaufen 
bekannten Fällen wies die Patientin keine der angeführten Risikofaktoren auf.

Der Nachweis einer Trichomoniasis der tiefen Atemwege kann grundsätzlich durch Sputumuntersuchungen geführt werden, allerdings mit eingeschränkter klinischer Wertigkeit. Die bronchoalveoläre Lavage erbrachte im vorliegenden Fall den Nachweis der Trichomonaden. Der Erregernachweis gemeinsam mit der Chronizität und dem klinischen Ansprechen der antiparasitären Therapie bestätigte indirekt die Diagnose der pulmonalen Trichomoniasis. Serologische Untersuchungen sind aufgrund der Kreuzreaktionen zwischen verschiedenen Trichomonas-Arten und der hohen Prävalenz der urogenitalen Trichomoniasis ohne diagnostischen Wert. Therapeutisches Mittel der Wahl ist Metronidazol.

Die pulmonale Trichomoniasis ist eine äußerst seltene Ursache bronchopulmonaler Infektionen, die möglicherweise zu selten diagnostiziert wird, da das Schrifttum nur spärliche Hinweise liefert. Der Krankheitsverlauf bei unserer Patientin verdeutlicht, daß es sich wahrscheinlich nicht um eine simple Kontamination, sondern um eine klinisch relevante Infektion mit Trichomonaden gehandelt hat. Wie häufig in der Medizin muß der endgültige Beweis der Kausalität letztlich offen bleiben.

\section{Literatur}

${ }^{1}$ Hersh SM. Pulmonary trichomoniasis and Trichomonas tenax. J Med Microbiol 1985; 20: 1 - 10

${ }^{2}$ El Kamel A, Rouetbi N, Chakroun M, Battikh M. Pulmonary eosinophilia due to Trichomonas tenax. Thorax 1996; 51: $554-$ 555

${ }^{3}$ Osborne PT, Giltman LI, Uthman EO. Trichomonads in the respiratory tract. A case report and literature review. Acta Cytol 1984; $28: 136-138$

${ }^{4}$ Memik F. Trichomonads in pleural effusion. JAMA 1968; 204 : 1145 - 1146

${ }^{5}$ Abed L, Delemotte J, Marill R, Ripert C, Tordjman G. [Pleuropulmonary localization of Trichomonas]. Bull Soc Pathol Exot Filiales 1966; 59: 962 - 964

${ }^{6}$ Shiota T, Arizono N, Morimoto T, Shimatsu A, Nakao K. Trichomonas tenax empyema in an immunocompromised patient with advanced cancer. Parasite 1998; 5: 375 - 377

${ }^{7}$ Walton BC, Bacharach T. Occurrence of Trichomonas in the respiratory tract. Report of three cases. J Parasitol 1963; 49: 35 38

${ }^{8}$ Skipina LV. [On trichomonads in the lungs]. Vrach Delo 1968; 4 : $134-135$

${ }^{9}$ Honigberg BM. Trichomonads found outside the urogenital tract of humans. In: Honigberg BM (ed). Trichomonads parasitic in humans. New York: Springer Verlag; 1990. 342 - 393

${ }^{10}$ Tumka AF. Trichomonal invasion of the lung (zitiert in 1). Klinicheskaia Meditsina 1956; 34: 35 - 40

${ }^{11}$ Teras IK, Ryigas EM, Kazakova II, Ranne KP, Trapido LE. [Detection of Trichomonas in the bronchi, sputum and oral cavity in various lung diseases]. Ter Arkh 1980; 52: 123 - 125

${ }^{12}$ Vrablic J, Catar G, Stanik R, Hromada J, Zitnan D. [Occurrence of the oral flagellate, Trichomonas tenax, in children with chronic tonsillitis]. Bratisl Lek Listy 1987; 88: 64 - 70

${ }^{13}$ Duboucher C, Mogenet M, Perie G. Salivary trichomoniasis. A case report of infestation of a submaxillary gland by Trichomonas tenax. Arch Pathol Lab Med 1995; 119: 277 - 279

${ }^{14}$ Mahdi NK, al-Saeed AT. Trichomonas tenax in Basrah, Iraq. J Pak Med Assoc 1993; 43: 261 - 262
${ }^{15}$ Vrablic J, Tomova S, Catar G, Randova L, Suttova S. [Morphology and diagnosis of Entamoeba gingivalis and Trichomonas tenax and their occurrence in children and adolescents]. Bratisl Lek Listy 1991; 92: $241-246$

Dr. med. Susanne M. Lang

Schwerpunkt Pneumologie

Medizinische Klinik

Klinikum Innenstadt

Ziemssenstr. 1

80336 München

E-mail: slang@medinn.med.uni-muenchen.de 